\title{
Analysis of Contributions of Car Body Sealing System to Vehicle Interior Aerodynamic Noise
}

\author{
Yinzhi He ${ }^{\mathrm{a}}$, Chunyang $\mathrm{Lu}^{\mathrm{b}}$ \\ Shanghai Automotive Wind Tunnel Center, Tongji University, Shanghai 201804, China \\ aheyinzhi@tongji.edu.cn, bluchunyang@tongji.edu.cn
}

Keywords: Interior aerodynamic noise, Wind tunnel test, Car body sealing, Leak noise.

Abstract. A full scale aero-acoustic wind tunnel test on a 4-door middle class sedan has been applied to determine contributions of major seal components to interior leak noise with "buildup" scheme. Results using psychoacoustic metrics loudness and articulation index (AI) were also compared. The results show that seals for doors, rearview mirrors and side windows are the most important leak noise sources for that vehicle. While selecting the method of analyzing seal contributions to interior noise, besides A-weighted sound pressure level (SPL), quality metrics such as loudness and AI should be considered as well.

\section{Introduction}

The main sources of vehicle interior noise are powertrain, road-tire and aerodynamic noise (wind noise). As engine, road-tire and other automobile noise has been reduced over the last years and as wind speed increases, aerodynamic noise has more contribution to vehicle interior noise relatively $[1,2]$. Therefore, wind noise has become an important factor that influences the acoustical environment comfort, drive comfort and safety, speech intelligibility and the possibility to identify exterior signals. The theoretical research of aerodynamic noise is not fully capable to be applied practically yet, however, experiment is intuitive and reliable. Thus, the full scale aero-acoustic wind tunnel test has become one of the most important measures to investigate aerodynamic noise.

This paper firstly presents a brief introduction of interior aerodynamic noise generation mechanism, especially leak noise generated by car body sealing system. Based on this theory, a full scale aero-acoustic wind tunnel test on a 4-door middle class sedan has been applied to determine the contributions of major seal components to interior leak noise with "buildup" scheme [3]. Additionally, the results were analyzed and compared with psychoacoustic metrics, i.e. loudness and AI.

\section{Effect of Body Sealing System on Interior Aerodynamic Noise}

Wind noise inside the cabin can be divided into two categories by generation and transmission mechanism. One is generated around vehicle surface due to external unsteady pressure fluctuations, and this kind of noise can be transmitted into passenger compartment through structures. The other is through leakage of air-flow into the cabin [4]. The latter is referred to as aspiration, or leak noise, which belongs to monopole source with a high efficiency of sound radiation. The problem mainly comes from a poor seal or a seal failure caused by body design, manufacturing or assembly processes. Typical scenario is that the pressure difference in the area of doors and side windows is large due to the air-flow over the vehicle surface, resulting in a leakage and then a monopole source generated. Furthermore, a leakage is a direct sound transmission path with a relative low transmission loss. Some research work has showed that if an aspiration noise path exists near an occupant's ear, it can dominate other wind noise sources [1]. Therefore, leak noise is the main research object in this paper.

\section{Test Method and Measurement System}

Test Platform. The test was carried out in the full scale aeroacoustic wind tunnel of Shanghai Automotive Wind Tunnel Center (SAWTC). This wind tunnel is equipped with a $3 / 4$ open-jet, closed 
test section with nozzle area $27 \mathrm{~m}^{2}$. The maximum wind speed can reach $250 \mathrm{kph}$. Background noise measured out-of-flow is less than $61 \mathrm{dBA}$ at the wind speed $160 \mathrm{kph}$ and it is one of the quietest wind tunnels worldwide [3]. The test vehicle was fixed on the middle part of the turntable during the test. Measurement System. Noise and vibration measurement and analysis system from HEAD Acoustics $\mathrm{GmbH}$ were used in this test, which comprises four HMS III digital artificial heads, multi-channel front-end SQlab III, binaural signal acquisition software HEAD Recorder and Artemis 9 analysis software. Four artificial heads were placed seperately in the driver's seat, co-driver's seat, the rear left and right passenger seat, which were used to collect binaural wind noise signal. Test cables were arranged through the rear seat and trunk into the interface box inside the wind tunnel turntable, and then went through the balance base into the data acquisition and analysis system in control room.

Test Method. Leak noise from different seal components has different contributions to the total interior noise, which were determined in the test with "buildup" scheme. First of all, outer parts and slot joints of the test vehicle were taped with sealing tape, as if all leakage "windows" on the body surface were closed, making the interior noise level at the most "quiet" state. Then the tapes on different seal components were uncovered in turn. It seems like opening the "windows" again. By comparing the test results with or without sealing tape, the contribution of different seal components to interior noise can be ranked and compared.

\section{Test Conditions and Procedure}

Condition 1. Outer parts and slot joints of the test vehicle were all taped with sealing tape, making the interior noise level at the most "quiet" state.

Condition 2-7. On the basis of condition 1, the tape on four doors, four side windows, four door handles, two rear triangular windows, sunroof and rearview mirrors sealing area were torn off one by one and the wind noise level of the test vehicle was tested under each condition.

Condition 8. All the tapes were torn off, and the test vehicle was at baseline condition. Then the wind noise level was tested again under the baseline condition.

All the conditions above were at the speed of $160 \mathrm{~km} / \mathrm{h}$ with yaw angle zero. Ambient temperature was 22 degrees Celsius, ambient humidity was $48.8 \%$, and ambient air pressure was $102.3 \mathrm{kpa}$.

\section{Analytical Methods.}

The total leak noise can be obtained by subtracting test result of condition 1 from condition 8 . As for leak noise from different seal components, it can also be obtained by subtracting test result of condition 1 from condition 2-7.

In most cases, the interior noise is quantified using A-weighted SPL. However, a number of psychoacoustic metrics can be selected to evaluate sound quality [5], such as loudness, AI, sharpness, harshness, fluctuation, etc. Among these metrics, only loudness analysis is widely studied and approved by ISO.

Below the results were analyzed using three different analytical methods, i.e. A-weighted SPL, loudness and AI analysis, in which only the magnitude of A-weighted SPL can be directly subtracted, so by comparing with condition 1 , the relative contribution can also be obtained.

A-weighted SPL. A-weighted SPL refers to the sound pressure level measured in A-weighted networks in decibels, denoted dB (A). A-weighted SPL is used to simulate the reaction of human ear on frequency characteristics of low-intensity noise under $55 \mathrm{~dB}$, equivalent to correct the frequency response with the inverted 40-phon-equal-loudness contour, in order to achieve frequency weighting. A-weighted SPL reflects the subjective perception of human ear to noise correctly, so it is the most commonly used noise evaluation indicators in ISO and many countries [6,7].

Loudness. Loudness, related closely to sound strength, depends mainly on sound signal frequency. Stevens [8] and Zwicker [9] have studied the loudness theory thoroughly, and fully considered human 
ear listening perception and noise masking effect. The results of their studies were both included in ISO532-1975 [10]. In Stevens' method, octave band is taken as analysis bandwidth, and it's suitable for diffuse field. However, in Zwicker's method, 1/3 octave band is taken for bandwidth analysis, which is suitable for either free-field or diffuse-field.

The signal analysis results of both methods are quite consistent with each other at $1000 \mathrm{~Hz}$, but there is relative huge difference at higher and lower frequency. Stevens' algorithm has its limitations in the scope of calculation, so it is not accurate enough for some signals. In the application frequency range, non- $1000 \mathrm{~Hz}$ frequency components has a deviation of 1-4sones comparing to Zwicker's method [11], so Zwicker's method was chosen in this paper.

AI. AI describes the speech intelligibility in the presence of noise. With an analysis frequency range of $200 \sim 6300 \mathrm{~Hz}, \mathrm{AI}$ is dramatically affected by background noise frequency and sound pressure level. In particular, the one-third octave spectrum of the background noise in the speech band has a significant effect on conversations. The overall AI is obtained by averaging the percentage of intelligibility for each frequency band. The biggest difference between AI and the first two methods is that the higher the AI, the quieter the passenger compartment.

\section{Analysis Results.}

Contribution Analysis of Seal Components to Interior Leakage Noise Using A-weighted SPL. Figure 1 is the A-weighted SPL histogram at the speed of $160 \mathrm{~km} / \mathrm{h}$, based on the test results at the outboard ear position of the driver's seat under different conditions. The seal components' contribution to interior leak noise was presented with overall sound pressure level (OASPL). As shown in Figure 1, for this test vehicle, among the main leak noise sources, contribution of doors, rearview mirrors, outer beltline weather strips, door handles, glass run seals, rear triangular windows and sunroof decrease successively. Contribution of doors, windows and rearview mirrors is more significant, while the contribution of sunroof and rear triangular window is relatively smaller.

Figure 2 to Figure 6 show respectively the A-weighted one-third octave spectrum of doors, rearview mirrors, outer beltline weather strips, door handles and glass run seals. The contribution of rear triangular windows and the sunroof is very small, so that the two lines in the spectrum are almost coincident in the whole frequency band. Therefore, the spectrums of those two cases are not given.

It can be seen from Figure 2 that in characteristic frequency band from about 200 to $10000 \mathrm{~Hz}$, door seals have an average contribution of about $2 \mathrm{~dB}$ (A). Figure 3 shows the rearview mirrors' characteristic frequency bands are $160 \sim 400 \mathrm{~Hz}$ and $4000 \sim 6300 \mathrm{~Hz}$, and the corresponding average contribution is about $1 \mathrm{~dB}(\mathrm{~A})$ and $1.7 \mathrm{~dB}(\mathrm{~A})$, which indicates that the rearview mirrors have good wind noise performance. Figure 4 shows that the outer beltline weather strips have a characteristic frequency band from around 1600 to $10000 \mathrm{~Hz}$, and the average contribution is about $2.4 \mathrm{~dB}$ (A). Figure 5 shows the characteristic frequency bands of door handles are $1600 \sim 2500 \mathrm{~Hz}$ and $5000 \sim$ $10000 \mathrm{~Hz}$, and the corresponding average contribution is about $1.1 \mathrm{~dB}(\mathrm{~A})$ and $2.1 \mathrm{~dB}(\mathrm{~A})$. Figure 6 shows that the characteristic frequency band of glass run seals is $1000 \sim 10000 \mathrm{~Hz}$ with an average contribution about $1.3 \mathrm{~dB}(\mathrm{~A})$.

The results above indicate that interior leakage noise mainly comes from door sealing (including door handles), rearview mirrors and side window seals (including glass run seals and outer beltline weather strips). In addition, the spectral characteristics of different noise sources are also different. 


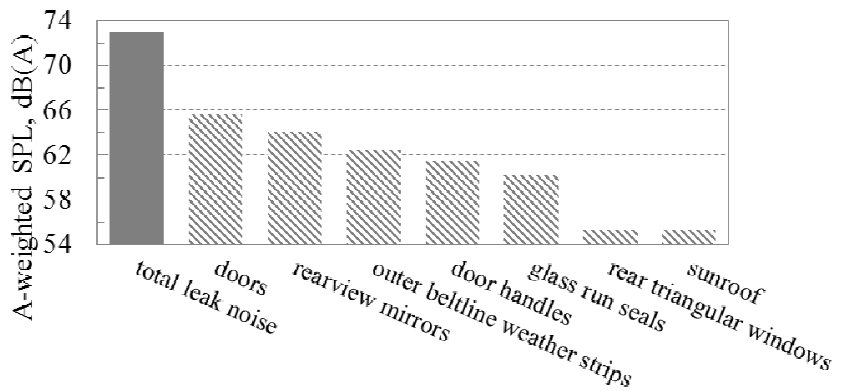

Figure 1. Contribution of seal components to interior leak noise at outboard ear of the driver's seat using A-weighted OASPL.

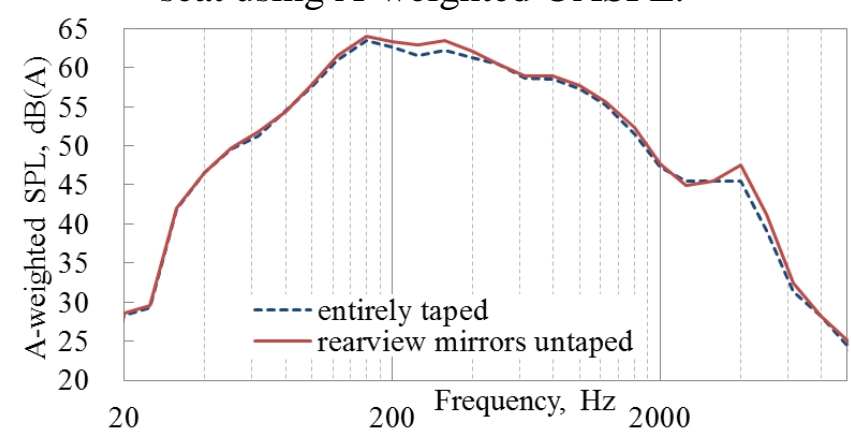

Figure 3. Characteristic spectrum under rearview mirrors taped/untaped condition.

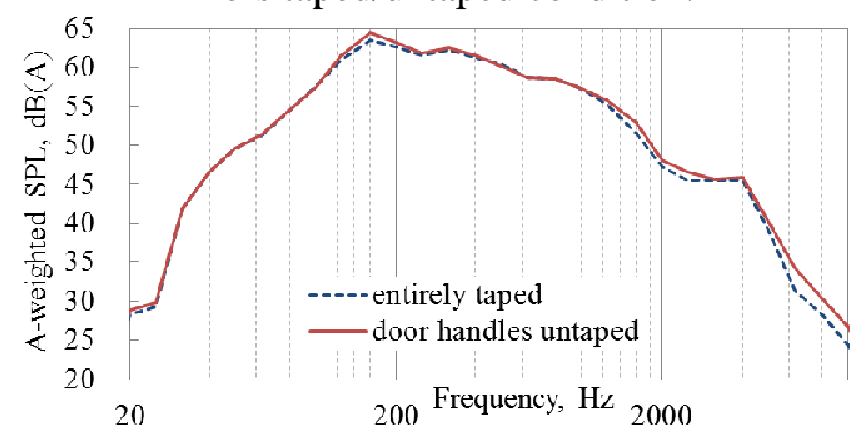

Figure 5. Characteristic spectrum under door handles taped/untaped condition.

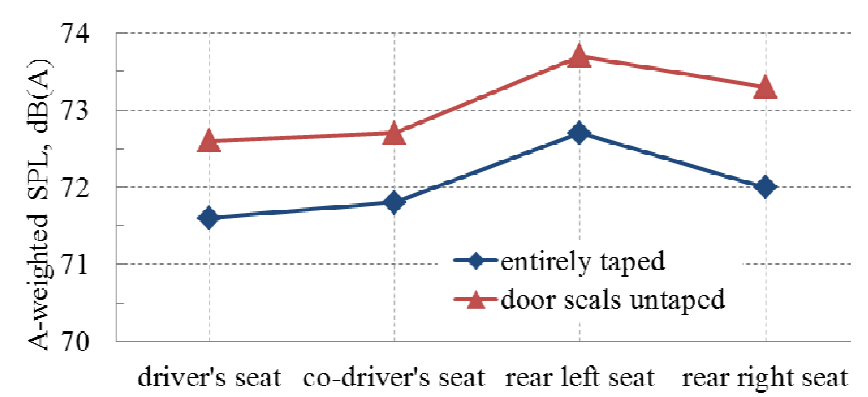

Figure 7. A-weighted OASPL of four outboard ears under doors taped/untaped conditions.

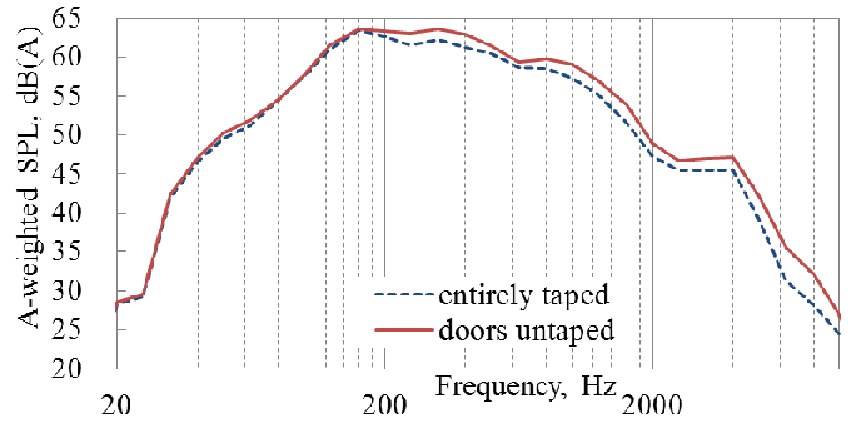

Figure 2. Characteristic spectrum under doors taped/untaped condition.

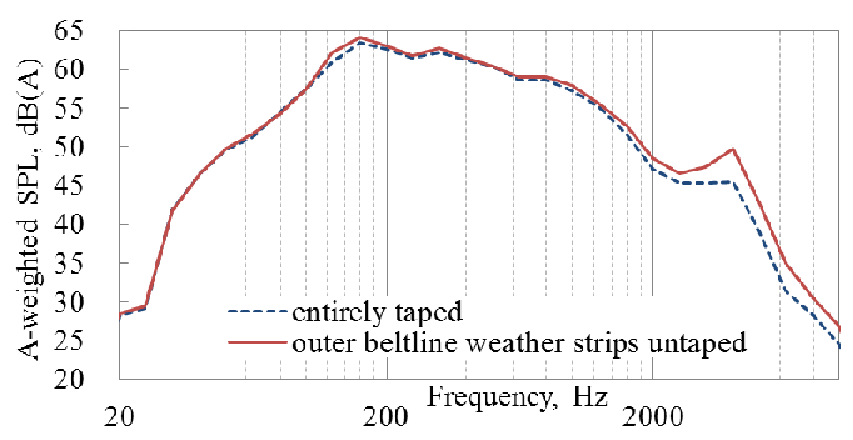

Figure 4. Characteristic spectrum under outer beltline weather strips taped/untaped condition.

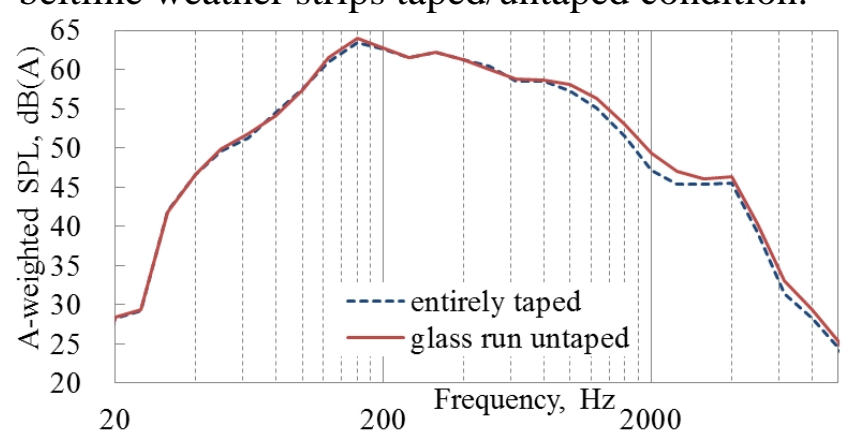

Figure 6. Characteristic spectrum under glass run taped/untaped condition.

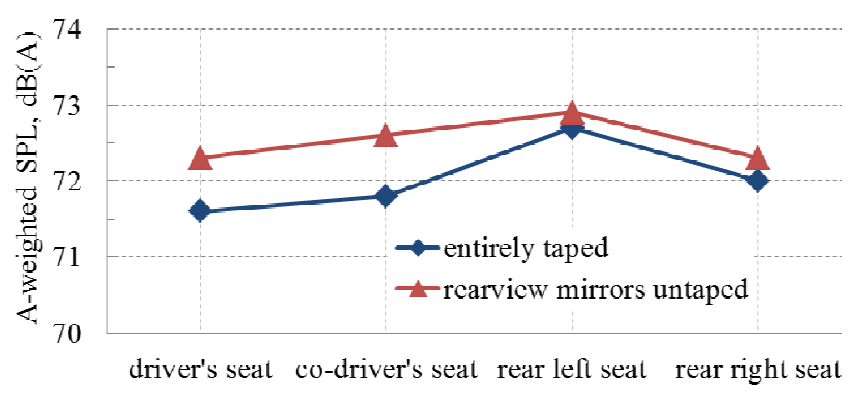

Figure 8. A-weighted OASPL of four outboard ears under rearview mirrors taped/untaped conditions.

Using the A-weighted OASPL measured at four outboard ear positions, Figure 7 and 8 show the influence of different leak noise sources to interior noise at different locations respectively. It can be seen clearly from the spacing between two polylines that the door seals' contribution is still the 
largest, followed by the rearview mirrors. As Figure 8 shows, the rearview mirror sealing has much more impact on the front two outboard ears than the rear two ears. The reason is probably that the rearview mirrors are located very close to the front outboard ears. Hence the leak noise from them can influence driver's position much more than that of rear seats.

Contribution Analysis of Seal Components to Interior Leakage Noise Using Loudness Analysis. As shown in Figure 9, for this test vehicle, among the main leak noise sources, contribution of doors, outer beltline weather strips, rearview mirrors, glass run seals, door handles, sunroof and rear triangular windows decrease successively. Contribution of doors, windows and rearview mirrors is relatively significant, while the contribution of sunroof and rear triangular window is relatively smaller. In general, this result and the A-weighted OASPL result are similar, i.e. door seals have the greatest contribution, the second and third are outer beltline weather strips and rearview mirrors, the fourth and fifth are glass run and door handles, the last two are rear triangular windows and sunroof.

As shown in Figure 9, at the wind speed of 160kph, based on condition 1(entirely taped), only the tape on door seals is torn off, the loudness at the outboard ear of the driver's seat increases by 2.3 sone; only the outer beltline weather strips are untaped, the loudness increases by 1.6sone; only the rearview mirrors are untaped, the loudness increases by 1.1 sone; only the glass run seals are untaped, the loudness increases by 0.9 sone; only the door handles are untaped, the loudness increases by 0.8 sone; only the sunroof is untaped, the loudness increases by 0.1 sone. However, when only the rear triangular windows are untaped, the loudness at the outboard ear of the driver's seat basically doesn't change.

To decrease the interior leak noise, doors (including door handles), rearview mirrors and window seals should be considered at first from above results. In this case, the loudness analysis is consistent with the A-weighted SPL analysis to some extent. It should also be noted that the analysis above is only for the signals measured at outboard ear of the driver's position.

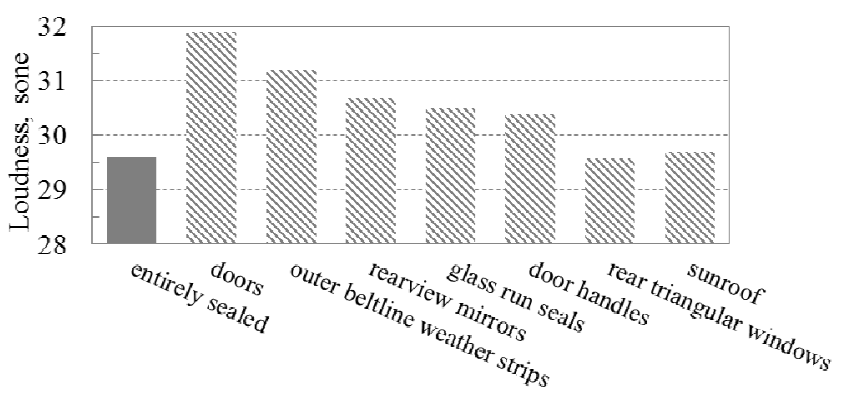

Figure 9. Contribution of seal components to interior leak noise at outboard ear of the driver's seat using Loudness analysis.

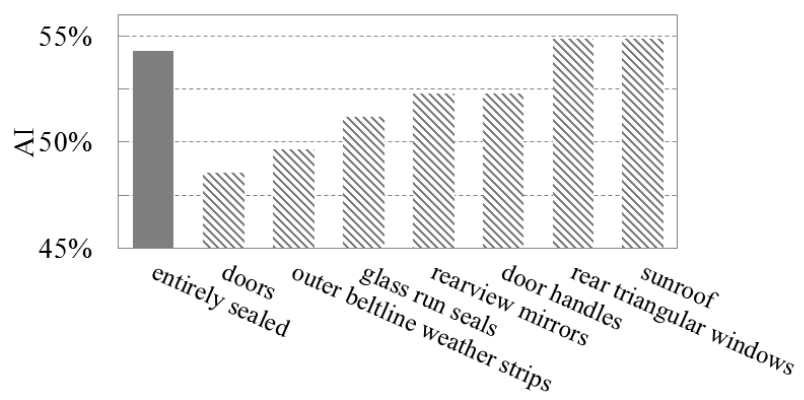

Figure 10. Contribution of seal components to interior leak noise at outboard ear of the driver's seat using AI analysis.

Contribution Analysis of Seal Components to Interior Leakage Noise Using AI Analysis. When AI analysis is used, it should be noted, the higher the AI, the better the sound quality. As shown in Figure 10, based on the fully taped condition, only the door seal is torn off, which leads to a decrease of AI at the outboard ear of driver's position by $5.7 \%$ as the maximum contribution; followed by outer beltline weather strips with $4.6 \%$; the third is the glass run seal, reducing the AI by $3.1 \%$; the rearview mirrors is at the fourth place, along with door handles, reducing the AI by $2.0 \%$. The interior AI basically doesn't change, when the tape on the rear triangular windows or sunroof is torn off.

The relative contribution by AI analysis is basically the same as loudness analysis, that is, the ranking is almost the same, except for the position exchange of the rearview mirrors and glass run. In contrast, the consistency between results of AI and A-weighted OASPL is relatively low. 


\section{Conclusions}

After the test vehicle was entirely taped, compared to the baseline condition, the spectral characteristics of interior noise changes significantly and the noise level decreases significantly as well. The sealing parts of doors, rearview mirrors and side windows (including glass run seals and beltline weather strips) are the areas where the leak noise is more serious, which could be the main consideration to improve interior wind noise performance.

As for analysis methods, A-weighted SPL is the most commonly used evaluation method, and the results of loudness analysis and AI are in good agreement. Although A-weighted SPL analysis has some limitations, the results still have reference value for sound quality in general. Sound quality evaluation with AI is different from A-weighted SPL and loudness, which is more sensitive to working condition changes. AI analysis frequency range is $200 \sim 6300 \mathrm{~Hz}$, which is only a subset of the normal noise analysis frequency range $(20 \sim 10000 \mathrm{~Hz})$, hence the difference of AI and A-weighted SPL analysis results may come from the frequency range difference.

In general, to analyze contribution of the body sealing system to interior aerodynamic noise, besides A-weighted SPL analysis, sound quality metrics such as loudness and AI should be considered as well.

\section{Acknowledgements}

This work was financially supported by the National Natural Science Foundation of China (51575394).

\section{References}

[1] George, A. R.: International Congress and Exposition. SAE Paper, 1990-03-15.

[2] Pang Jian, Gang Chen, Hua He: Automobile Noise and Vibration: Theory and Application. (Beijing Institute of Technology Press, Beijing, 2006).In Chinese.

[3] Yinzhi He, Zhigang Yang, Yigang Wang: submitted to Journal of Automotive Engineering (2012). In Chinese.

[4] Y. Gur, K.N. Morman, N. Singh: SAE 1997 Noise \& Vibration Conference \& Exposition.

[5] Kousuke Noumura, Junji Yoshida: SAE 2003 Noise \& Vibration Conference \& Exhibition.

[6] Mingze Zhang: submitted to Journal of Technical Acoustics (1995). In Chinese.

[7] Qinglin Li, Changjing Ye, Runcheng Kang: submitted to Auto SCI-TECH (2013). In Chinese.

[8] Stevens S.S.: submitted to Journal of the Acoustical Society of America (1956).

[9] Zwicker E., Scharf B.: submitted to Psychological Review (1965).

[10] ISO 532 - 1975 Acoustic-method for calculation loudness level. 1975.

[11] Feng Chen: Study on the Evaluation Method of Psychoacoustics Loudness. (Master Thesis, Huazhong University of Science and Technology, 2009). In Chinese. 